\title{
On the Perron Effect for Exponential Stability of Differential Systems on Time Scales
}

\author{
Le Duc Nhien", Le Huy Tien \\ Department of Mathematics, Mechanics and Informatics, VNU University of Science, \\ 334 Nguyen Trai, Thanh Xuan, Hanoi, Vietnam
}

Received 02 September 2017

Revised 29 September 2017; Accepted 24 October 2017

\begin{abstract}
In 2007, N. H. Du and L. H. Tien [1] shown that the exponential stability of the linear equation on time scales implies the exponential stability of the suitable small enough Lipchitz perturbed equation. In this paper, we shall prove that if the perturbation is arbitrary small order 1 then the above argument is not true which is called Perron effect.
\end{abstract}

Keywords: Exponential stability, Perron effect, time scales, linear dynamic equation.

\section{Introduction and preliminaries}

Theory of dynamic equations on time scales was introduced by Stefan Hilger [2] in order to unify and extend results of differential equations, difference equations, $q$-difference equations, etc. There are many works concerned with the stability of dynamic equations on time scales such as exponential stability (see [3-5]); dichotomies of dynamic equations (see [6]).

In this paper, we want to go further in the stability of dynamic equations. More precisely, we show that the exponential stability of the linear equation on time scales does not imply the exponential stability of the small enough Lipchitz perturbed equation if the perturbation is arbitrary small order 1 which is called Perron effect. Moreover, our results are different from examples of Perron type in both continuous and discrete cases (see [7-9]).

We now introduce some basic concepts of time scales, which can be found in [10,11]. A time scale $\mathbb{T}$ is defined as a nonempty closed subset of the real numbers. Define the forward jump operator $\sigma: \mathbb{T} \rightarrow \mathbb{T}$ is defined by $\sigma(t)=\inf \{s \in \mathbb{T}: s>t\}$ and the graininess function $\mu(t)=\sigma(t)-t$ for any $t \in \mathbb{T}$. In the following discussion, the time scale $\mathbb{T}$ is assumed to be unbounded above and below. We have the following several basis definitions (see [10, 11]).

\footnotetext{
* Corresponding author. Tel.: 84-1683523242.

Email: nhien0610@gmail.com

https//doi.org/ 10.25073/2588-1124/vnumap.4230
} 
Definition 1.1. Let $A$ be an $m \times n$ matrix-valued function on $\mathbb{T}$. We say that $A$ is $r d$-continuous on $\mathbb{T}$ if each entry of $A$ is rd-continuous on $\mathbb{T}$, and the class of all such $r d$-continuous $m \times n$ matrixvalued funtions on $\mathbb{T}$ is denoted by

$$
C_{r d}=C_{r d}(\mathbb{T})=C_{r d}\left(\mathbb{T}, \mathbb{R}^{m \times n}\right) .
$$

We say that $A$ is differentiable on $\mathbb{T}$ provided each entry of $A$ is differentiable on $\mathbb{T}$, and in this case we put

$$
A^{\Delta}=\left(a_{i, j}^{\Delta}\right)_{l \leq i \leq m, l \leq j \leq n} \text { where } A=\left(a_{i, j}\right)_{1 \leq i \leq m, l \leq j \leq n} .
$$

Definition 1.2 (Regressivity). An $n \times n$ matrix-valued function $A$ on a time scale $\mathbb{T}$ is called regressive (with respect to $\mathbb{T}$ ) provided

$I+\mu(t) A(t)$ is invertible for all $t \in \mathbb{T}^{\kappa}$,

and the class of all such regressive function is denoted

$$
\mathcal{R}=\mathcal{R}(\mathbb{T})=\mathcal{R}\left(\mathbb{T}, \mathbb{R}^{n \times n}\right) .
$$

Throughout this paper we only consider $A(t) \in \mathcal{R} \cap C_{r d}$.

Definition 1.2. Assume $A$ and $B$ are regressive $n \times n$ matrix-valued functions on $\mathbb{T}$. Then we define $A \oplus B$ by

$(A \oplus B)(t)=A(t)+B(t)+\mu(t) A(t) B(t)$ for all $t \in \mathbb{T}^{\kappa}$,

and we define $\odot A$ by

$(\ominus A)(t)=-A(t)[I+\mu(t) A(t)]^{-1}$ for all $t \in \mathbb{T}^{\kappa}$.

Remark 1.1. $\left(\mathcal{R}\left(\mathbb{T}, \mathbb{R}^{n \times n}\right), \oplus\right)$ is a group.

Definition 1.4 (Matrix Exponential Function). Let $t_{0} \in \mathbb{T}$ and assume that $A \in \mathcal{R}$ is an $n \times n$ matrix-valued function. The unique matrix-value solution of the IVP

$$
x^{\Delta}=A(t) x, \quad x\left(t_{0}\right)=I,
$$

where I denotes as usual the $n \times n$ identity matrix, is called the matrix exponential function (at $\left.t_{0}\right)$, and it is denoted by $e_{A}\left(\cdot, t_{0}\right)$.

We collect some fundamental properties of the exponential function on time scales.

Theorem 1.1 (see [10]). If $A, B \in \mathcal{R}$ are matrix-valued function on $\mathbb{T}$, then

(i) $e_{0}(t, s) \equiv I$ and $e_{A}(t, t) \equiv I$,

(ii) $e_{A}(\sigma(t), s)=(I+\mu(t) A(t)) e_{A}(t, s)$,

(iii) $e_{A}(t, s)=\left[e_{A}(s, t)\right]^{-1}=\left[e_{\odot A^{*}}(s, t)\right]^{*}$,

(iv) $e_{A}(t, s) e_{A}(s, \tau)=e_{A}(t, \tau)$,

(v) $e_{A}(t, s) e_{B}(t, s)=e_{A \oplus B}(t, s)$ if $e_{A}(t, s)$ and $B(t)$ commute.

If $n=1$, one have the equivalent definition of the exponential function on time scales by 


$$
e_{p}(t, s)=\exp \left\{\int_{s}^{t} \xi_{\mu(\tau)}(p(\tau)) \Delta \tau\right\}
$$

with

$$
\xi_{h}(z)=\lim _{u \downarrow h} \frac{\log (1+u z)}{u}=\left\{\begin{array}{cl}
z & \text { if } h=0 \\
\log (1+h z) / h & \text { if } h \neq 0
\end{array} .\right.
$$

For any $p \in \mathcal{R}(\mathbb{T}, \mathbb{R})$ and $s, t \in \mathbb{T}$, where $\log$ is principal logarithm. It is easy that with $z \in \mathbb{R}$, the inverse transformation of $\xi_{h}$ is given by

$$
\xi_{h}^{-l}(z)=\lim _{u \downarrow h} \frac{e^{u z}-1}{u}==\left\{\begin{array}{cl}
z & \text { if } h=0 \\
\frac{1}{h}\left(e^{z h}-1\right) & \text { if } h \neq 0
\end{array} .\right.
$$

We refer [10] and [11] for more information on analysis on time scales. From now on, we fix a $t_{0} \in \mathbb{T}, t_{0} \geq 1$ and denote $\mathbb{T}^{+}:=\left[t_{0},+\infty\right)$ with the graininess of underlying time scale is bounded on $\mathbb{T}^{+}$, i.e., $M=\sup _{t \in \mathbb{T}^{+}} \mu(t)<\infty$. Besides, considered time scales are always upper unbounded, i.e., for all $n \in \mathbb{N}$, there exists $t_{n} \in \mathbb{T}, t_{n} \geq n$. We consider a dynamic equation on time scale $\mathbb{T}$

$$
x^{4}(t)=F(t, x), \quad t \in \mathbb{T}^{+},
$$

where $F(t, x): \mathbb{T}^{+} \times \mathbb{R}^{n} \rightarrow \mathbb{R}^{n}$ is rd-continuous in the first variable with $F(t, 0)=0$. We also suppose that $F$ satisfies all conditions such that (2) has a unique solution $x(t)$ with $x\left(t_{0}\right)=x_{0}$ on $\left[t_{0},+\infty\right)$.

The following definition is a concept of exponential stability as in [1].

\section{Definition 1.5.}

(i) The solution $x=0$ of Eq. (2) is said to be exponentially stable if there exists a positive constant $\alpha$ with $-\alpha \in \mathcal{R}^{+}$such that for every $\tau \in \mathbb{T}^{+}$, there exists $N=N(\tau) \geq 1$ such that the solution of (2) through $(\tau, x(\tau))$ satisfies

$\|x(t)\| \leq N\|x(\tau)\| e_{-\alpha}(t, \tau) \quad$ for all $t \geq \tau, t \in \mathbb{T}^{+}$.

(ii) The solution $x=0$ of Eq. (2) is said to be uniformly exponentially stable if it is exponentially stable and constant $N$ can be chosen independently of $\tau \in \mathbb{T}^{+}$.

We now consider the perturbed equation of equation (1)

$$
x^{\Delta}(t)=A(t) x(t)+f(t, x), \quad t \in \mathbb{T}^{+},
$$

where $f(t, x): \mathbb{T}^{+} \times \mathbb{R}^{n} \rightarrow \mathbb{R}^{n}$ is rd-continuous in the first argument with $f(t, 0)=0$.

The following theorem is well known, due to Du and Tien et all. [1]

Theorem 1.2. If the following conditions are satisfied

(i) Equation (1) is exponentially stable with constants $\alpha$ and $N$,

(ii) $\|f(t, x)\| \leq L\|x\|$ for all $t \in \mathbb{T}^{+}$,

(iii) $\alpha-N L>0$, 
then the solution $x=0$ of Eq. (3) is exponentially stable.

We have a natural question that How the previous theorem is? if the conditions (ii) and (iii) of previous theorem are replaced by arbitrary small order 1 property where perturbation, say $f(t, x)$, is called arbitrary small order $\alpha$ if

$$
\lim _{x \rightarrow 0} \frac{\|f(t, x)\|}{\|x\|^{\alpha}}<\infty
$$

Denote $s(t)=t \sin \log t ; a, M$ and $b$ are positive constants satisfies $b<2$,

$$
H(M)<2 a<\frac{2}{2-b} \text { with } H(M)=\lim _{s \rightarrow M} \frac{e^{s(2+s)}-1}{s} .
$$

Remark 1.2. It is easy to check that $H(M) \geq 2^{1 / 2}$ for all $M \in[0,+\infty)$.

With previous conditions, we now give the main theorems in this paper which is the answer of the question.

Theorem 1.3. The trivial solution of the linear equation

$$
\left\{\begin{array}{l}
x_{1}^{\Delta}=((a b) \ominus(2 a)) x_{1} \\
x_{2}^{\Delta}=\left[\xi_{\mu(t)}^{-1}\left(s^{\Delta}(t)\right) \ominus(2 a)\right] x_{2}
\end{array}\right.
$$

is exponential stable.

Consider the perturbed equation

$$
\left\{\begin{array}{l}
x_{1}^{\Delta}=((a b) \ominus(2 a)) x_{1} \\
x_{2}^{\Delta}=\left[\xi_{\mu(t)}^{-1}\left(s^{\Delta}(t)\right) \ominus(2 a)\right] x_{2}+a b x_{2}+\mu(t) a b\left[\xi_{\mu(t)}^{-1}\left(s^{\Delta}(t)\right) \Theta(2 a)\right] x_{2}+x_{1}^{\sigma}
\end{array} .\right.
$$

The following theorem is Perron effect for the exponential stability of the linear equation on time scales with a perturbation are arbitrary small order 1.

Theorem 1.4. The trivial solution of Eq. (6) is not exponential stable.

It also should be noticed that in the case $\mathbb{T}=\mathbb{R}$ then the pair of equations (5) and (6) become

$$
\left\{\begin{array} { l } 
{ x _ { 1 } ^ { \prime } = a ( b - 2 ) x _ { 1 } } \\
{ x _ { 2 } ^ { \prime } = [ s ^ { \prime } ( t ) - 2 a ] x _ { 2 } }
\end{array} \text { and } \left\{\begin{array}{l}
x_{1}^{\prime}=a(b-2) x_{1} \\
x_{2}^{\prime}=\left[s^{\prime}(t)-2 a\right] x_{2}+a b x_{2}+x_{1}
\end{array}\right.\right.
$$

with $a, b$ satisfy $1<a<1 /(2-b)$, which is the differential example of the Perron's one (see [7, 8]). Besides, we also obtain the differential example in the difference case of N. V. Kuznetsov, G. A. Leonov ([9]) as the following pair

$$
\left\{\begin{array}{l}
x_{1}(n+1)=\frac{a b+1}{1+2 a} x_{1}(n) \\
x_{2}(n+1)=\frac{\exp (s(n+1)-s(n))}{1+2 a} x_{2}(n)
\end{array},\right.
$$

and 
$\left\{\begin{array}{l}x_{1}(n+1)=\frac{a b+1}{1+2 a} x_{1}(n) \\ x_{2}(n+1)=\frac{(a b+1) \exp (s(n+1)-s(n))}{1+2 a} x_{2}(n)+a b x_{2}(n)+x_{1}(n+1)\end{array}\right.$,

with $a, b$ satisfy $\left(e^{3}-1\right) / 2<a<1 /(2-b)$.

\section{Proof of main theorems}

This section is devoted to the proof of Theorem 1.3 and 1.4. We shall present these proof in two subsection.

Proof of Theorem 1.3. We first consider the solution $x(t)$ of Eq. (5) with initial condition $\left(x_{I}\left(t_{0}\right), 0\right), x_{I}\left(t_{0}\right) \neq 0$. Then $x$ has the form

$x(t)=\left(e_{(a b) \ominus(2 a)}\left(t, t_{0}\right) x_{1}\left(t_{0}\right), 0\right)$.

It is clear that

$$
\begin{aligned}
\|x\| & =e_{(a b) \in(2 a)}\left(t, t_{0}\right)\left|x_{I}\left(t_{0}\right)\right| \\
& =\exp \left\{\int_{t_{0}}^{t} \lim _{u \unlhd \mu(\tau)} \frac{1}{u} \log (1+((a b) \ominus(2 a)) u) \Delta \tau\right\}\left|x_{I}\left(t_{0}\right)\right| \\
& =\exp \left\{\int_{t_{0}}^{t} \lim _{u \unlhd \mu(\tau)} \frac{1}{u} \log \left(1+\frac{u(a b-2 a)}{1+2 a \mu(\tau)}\right) \Delta \tau\right\}\left|x_{I}\left(t_{0}\right)\right| \\
& \leq \exp \left\{\int_{t_{0}}^{t} \lim _{u \searrow \mu(\tau)} \frac{1}{u} \log \left(1+\frac{u a(b-2)}{1+2 a M}\right) \Delta \tau\right\}\left|x_{I}\left(t_{0}\right)\right| \quad(\text { since } b<2)
\end{aligned}
$$

Set $L=\frac{a(2-b)}{1+2 a M}>0$. The previous relation implies

$$
\|x\| \leq e_{-L}\left(t, t_{0}\right)\left|x_{I}\left(t_{0}\right)\right| \text {. }
$$

Second, we consider the solution with initial condition $\left(0, x_{2}\left(t_{0}\right)\right)$. Then the $x$ is given by

$$
x(t)=\left(x_{1}(t), x_{2}(t)\right)=\left(0, e_{\xi_{\mu}^{-l}\left(s^{4}\right) \in(2 a)}\left(t, t_{0}\right) x_{2}\left(t_{0}\right)\right) .
$$

Then, we have

$$
\begin{aligned}
& || x(t) \| e_{\xi_{\mu}^{-1}\left(s^{\Delta}\right) \ominus(2 a)}\left(t, t_{0}\right)\left|x_{2}\left(t_{0}\right)\right| \\
& =\exp \left\{\int_{t_{0}}^{t} \lim _{u\lrcorner \mu(\tau)} \frac{1}{u} \log \left(1+u\left(\xi_{\mu(\tau)}^{-1}\left(s^{\Delta}\right) \ominus(2 a)\right)\right) \Delta \tau\right\}\left|x_{2}\left(t_{0}\right)\right| \\
& =\exp \left\{\int_{t_{0}}^{t} \lim _{u \unlhd(\tau)} \frac{1}{u} \log \left(1+\frac{u\left(\xi_{\mu(\tau)}^{-1}\left(s^{\Delta}\right)-2 a\right)}{1+2 a \mu(\tau)}\right) \Delta \tau\right\}\left|x_{2}\left(t_{0}\right)\right|,
\end{aligned}
$$


where

$$
\xi_{\mu(\tau)}^{-1}\left(s^{\Delta}(\tau)\right)=\lim _{h \searrow \mu(\tau)} \frac{e^{h s^{\Delta}(\tau)}-1}{h}=\lim _{h \searrow \mu(\tau)} \frac{e^{h\left(\sin \log (\tau)+(\tau+\mu(\tau)) \cos \log c \cdot \log ^{\Delta}(\tau)\right)}-1}{h},
$$

with $c \in[\tau, \tau+\mu(\tau)]$ (by Theorem 1.87 in [10]). One have two following cases.

Case 1. If $\tau$ is right - scattered then

$$
\log ^{\Delta}(\tau)=\frac{\log (\tau+\mu(\tau))-\log \tau}{\mu(\tau)}=\frac{1}{\mu(\tau)} \log \left(1+\frac{\mu(\tau)}{\tau}\right) \leq \frac{1}{\tau} .
$$

Hence, from (9) we obtain

$$
\xi_{\mu(\tau)}^{-l}\left(s^{\Delta}(\tau)\right) \leq \frac{e^{\mu(\tau)(2+M / \tau)}-1}{\mu(\tau)} \leq \frac{e^{\mu(\tau)(2+M)}-1}{\mu(\tau)},
$$

if $\tau \geq t_{0} \geq 1$. Since $O \leq \mu(t) \leq M$ and $f(x)=\frac{e^{x(2+M)}-1}{x}$ is the increasing function on $(0, M]$ therefore

$$
\xi_{\mu(\tau)}^{-1}\left(s^{\Delta}(\tau)\right) \leq \frac{e^{M(2+M)}-1}{M}=H(M) .
$$

Case 2. If $\tau$ is right - dense then $\log ^{4}(\tau)=1 / \tau$. Thus, from (9) and Remark 1.2 we obtain $\xi_{\mu(\tau)}^{-I}\left(s^{\Delta}(\tau)\right)=\sin \log (\tau)+\cos \log (\tau) \leq \sqrt{2} \leq H(M)$.

By the hypothesis (4), $H(M)<2 a$, we deduce $\xi_{\mu(\tau)}^{-1}\left(s^{4}\right)-2 a<0$. Combining with (8), it implies

$$
\begin{aligned}
\|x(t)\| & \leq \exp \left\{\int_{t_{0}}^{t} \lim _{u \unlhd \mu(\tau)} \frac{1}{u} \log \left(1-\frac{u(2 a-H(M))}{1+2 M a}\right) \Delta \tau\right\}\left|x_{2}\left(t_{0}\right)\right| \\
& =e_{-(2 a-H(M)) /(1+2 M a)}\left(t, t_{0}\right)\left|x_{2}\left(t_{0}\right)\right| .
\end{aligned}
$$

From (7), (10) and the condition of $a, b$ and $M$ implies the trivial solution of Eq. (2) is exponential stable.

Proof of Theorem 1.4. From the first equation of system (5) we have

$$
x_{I}(t)=x_{I}\left(t_{0}\right) e_{(a b) \ominus(2 a)}\left(t, t_{0}\right) \text {. }
$$

Therefore, the second one becomes

$$
x_{2}^{\Delta}=\left[\xi_{\mu(t)}^{-1}\left(s^{\Delta}(t)\right) \ominus 2 a\right] x_{2}+a b x_{2}+\mu(t) a b\left[\xi_{\mu(t)}^{-1}\left(s^{\Delta}(t)\right) \ominus 2 a\right] x_{2}+\left[x_{1}\left(t_{0}\right) e_{(a b) \ominus(2 a)}\left(t, t_{0}\right)\right]^{\sigma} \text {. }
$$

By the variation of constants formula we have the solution of (11) has the form

$$
\begin{aligned}
& x_{2}(t)=x_{2}\left(t_{0}\right) e_{\left(\xi_{\mu}^{-l}\left(s^{4}\right) \ominus 2 a\right) \oplus(a b)}\left(t, t_{0}\right)+\int_{t_{0}}^{t} e_{\left(\xi_{\mu}^{-l}\left(s^{4}\right) \oplus 2 a\right) \oplus(a b)}(t, \sigma(\tau))\left[x_{I}\left(t_{0}\right) e_{(a b) \Theta(2 a)}\left(\sigma(\tau), t_{0}\right)\right] \Delta \tau \\
\Leftrightarrow & x_{2}(t)=x_{2}\left(t_{0}\right) e_{\left(\xi_{\mu}^{-l}\left(s^{4}\right) \oplus 2 a\right) \oplus(a b)}\left(t, t_{0}\right)+x_{I}\left(t_{0}\right) e_{\xi_{\mu}^{-l}\left(s^{\Delta}\right)}\left(t, t_{0}\right) e_{(a b) \ominus(2 a)}\left(t, t_{0}\right) \int_{t_{0}}^{t} e_{\xi_{\mu}^{-l}\left(s^{4}\right)}\left(t_{0}, \sigma(\tau)\right) \Delta \tau .
\end{aligned}
$$

Choosing $x_{2}\left(t_{0}\right)>0$, we deduce 


$$
x_{2}(t) \geq x_{I}\left(t_{0}\right) e_{\xi_{\mu}^{-l}\left(s^{\Delta}\right)}\left(t, t_{0}\right) e_{(a b) \ominus(2 a)}\left(t, t_{0}\right) \int_{t_{0}}^{t} e_{\xi_{\mu}^{-l}\left(s^{\Delta}\right)}\left(t_{0}, \sigma(\tau)\right) \Delta \tau .
$$

Moreover, by (3.3) in [12] and $(2 a) \ominus(a b)>0$ (since $b<2$ ), we have estimation

$$
\begin{aligned}
& \quad e_{(a b) \ominus(2 a)}\left(t, t_{0}\right)=e_{\Theta((2 a) \ominus(a b))}\left(t, t_{0}\right) \geq \exp \left\{-((2 a) \Theta(a b))\left(t-t_{0}\right)\right\} \\
& \geq \exp \left\{-\frac{(2 a-a b)}{1+2 a \mu(t)}\left(t-t_{0}\right)\right\} \\
& \geq \exp \left(a(b-2)\left(t-t_{0}\right)\right) .
\end{aligned}
$$

Since $\mathbb{T}$ is upper unbounded, there exists $t_{k} \in \mathbb{T}, t_{k}>e^{2 k \pi-\pi / 2}+1(k \in \mathbb{N})$. We consider

$$
\begin{aligned}
& I=e_{\xi_{\mu}^{-l}\left(s^{\Delta}\right)}\left(t_{k}, t_{0}\right) \int_{t_{0}}^{t_{k}} e_{\xi_{\mu}^{-l}\left(s^{\Delta}\right)}\left(t_{0}, \sigma(\tau)\right) \Delta \tau \\
& =\exp \left\{\int_{t_{0}}^{t_{k}} s^{\Delta}(\tau) \Delta \tau\right\} \int_{t_{0}}^{t_{k}} \exp \left\{\int_{\sigma(\tau)}^{t_{0}} s^{\Delta}(s) \Delta s\right\} \Delta \tau \\
& =\exp \left(s\left(t_{k}\right)-s\left(t_{0}\right)\right) \int_{t_{0}}^{t_{k}} \exp \left(s\left(t_{0}\right)-s(\sigma(\tau))\right) \Delta \tau \\
& =\exp \left(t_{k} \sin \ln t_{k}\right) \int_{t_{0}}^{t_{k}} \exp (-\sigma(\tau) \sin \log \sigma(\tau)) \Delta \tau .
\end{aligned}
$$

It is clear that when $k$ is large enough then

$$
I=\exp \left(t_{k}\right) \int_{t_{0}}^{t_{k}} \exp (-\sigma(\tau) \sin \log \sigma(\tau)) \Delta \tau .
$$

Since $t_{k}>e^{2 k \pi-\pi / 2}+1$, we get $\left[e^{2 k \pi-\pi / 2}, e^{2 k \pi-\pi / 2}+1\right] \subset\left[t_{0}, t_{k}\right]$ when $k$ is large enough. Hence,

$$
I \geq \exp \left(t_{k}\right) \int_{e^{2 k \pi-\pi / 2}}^{e^{2 k \pi-\pi / 2+1}} \exp (-\sigma(\tau) \sin \log \sigma(\tau)) \Delta \tau .
$$

In addition, by the relation between Lebesgue integration on $\mathbb{T}$ and $\mathbb{R}$ (see Theorem 5.2 in [13]), we get

$$
\begin{aligned}
& \exp \left(t_{k}\right) \int_{e^{2 k \pi-\pi / 2}}^{e^{2 k \pi-\pi / 2+1}} \exp (-\sigma(\tau) \sin \log \sigma(\tau)) \Delta \tau \\
& =\exp \left(t_{k}\right) \int_{e^{2 k \pi-\pi / 2}}^{e^{2 k \pi-\pi / 2+1}} \exp (-\sigma(\tau) \sin \log \sigma(\tau)) d \tau+\sum_{i \in I_{k}} \exp \left(-\sigma\left(a_{i}\right) \sin \log \sigma\left(a_{i}\right)\right) \mu_{\Delta}\left(a_{i}\right),
\end{aligned}
$$

where $a_{i} \in\left[e^{2 k \pi-\pi / 2}, e^{2 k \pi-\pi / 2+1}\right]$ with $\sigma\left(a_{i}\right)>0$ for all $i \in I_{k}$ and $\mu_{\Delta}$ is $\Delta$-measure on $\mathbb{T}$. It implies

$$
I \geq \exp \left(t_{k}\right) \int_{e^{2 k \pi-\pi / 2}}^{e^{2 k \pi-\pi / 2}+1} \exp (-\sigma(\tau) \sin \log (\sigma(\tau)) d \tau
$$




$$
=\exp \left(t_{k}\right) \int_{e^{2 k \pi-\pi / 2}}^{e^{2 k \pi-\pi / 2}+1} \exp (-(\tau+\mu(\tau)) \sin \log (\tau+\mu(\tau)) d \tau .
$$

When $k$ is large enough again then $\exp (-(\tau+\mu(\tau)) \sin \log (\tau+\mu(\tau)) \approx \exp (-\tau \sin \log \tau)$. Therefore,

$$
\begin{aligned}
& I=\exp \left(t_{k}\right) \int_{e^{2 k \pi-\pi / 2}}^{e^{2 k \pi-\pi / 2}+1} \exp (-\tau \sin \log \tau) d \tau \\
& \geq \exp \left(t_{k}\right) \int_{e^{2 k \pi-\pi / 2}}^{e^{2 k \pi / 2}+1} \exp \left(-\left(e^{2 k \pi-\pi / 2}+1\right) \sin \log \left(e^{2 k \pi-\pi / 2}+1\right)\right) d \tau \\
& =\exp \left(t_{k}\right) \exp \left(e^{2 k \pi-\pi / 2}\right) \geq \exp \left(t_{k}\right) .
\end{aligned}
$$

It means

$$
I \geq \exp \left(t_{k}-t_{0}\right) \quad\left(\text { when } t_{0}>1\right) \text {. }
$$

From (12), (13) and the last relation we obtain

$$
x_{2}\left(t_{k}\right) \geq x_{1}\left(t_{0}\right) \exp \left\{(a(b-2)+1)\left(t_{k}-t_{0}\right)\right\} \text {. }
$$

By hypothesis (4), we have $a(b-2)+1>0$, therefore

$$
\lim _{k \rightarrow \infty} x_{2}\left(t_{k}\right)=+\infty \text {. }
$$

It implies that the system (6) is unstable.

\section{Acknowledgments}

This research is funded by the VNU University of Science under project number TN.16.03. We would like to show our great thanks to the anonymous referee for his/her valuable suggestions and comments, which have improved a former version of this paper.

\section{References}

[1] N. H. Du and L. H. Tien, On the exponential stability of dynamic equations on time scales, J. Math. Anal. Appl, 331(2007), 1159 - 1174.

[2] S. Hilger, Analysis on measure chains - a unified approach to continuous and discrete calculus, Results Math. 18 (1990) 18--56.

[3] W. A. Coppel, Dichotomies in Stability Theory, Lecture Notes in Mathematics No. 29, Springer-Verlag, Berlin, 1978.

[4] A. Peterson, R. F. Raffoul, Exponential stability of dynamic equations on time scales, Adv. Difference Equ. Appl. (2005) 133--144.

[5] C. Potzsche, S. Siegmund and F. Wirth, A spectral characterization of exponential stability for linear timeinvariant systems on time scales, Discrete Contin. Dyn. Syst. 9 (2003) 1223-1241.

[6] C. Potzsche, Exponential dichotomies of linear dynamic equations on measure chains under slowly varying coefficients, J. Anal. Math. Appl. 289 (2004) 317-335.

[7] O. Perron, Die Stabilitatsfrage bei Differentialgleichungen, Mathematische Zeitschrift, 1930, vol. 32, no. 5, pp. $702-728$. 
[8] S. K. Korovin, N. A. Izobov, On the Perron Sign Change Effect for Lyapunov Characteristic Exponents of Solutions of Differential Systems, 2010, Diff. Equa, vol. 46, no. 10, pp. 1395 - 1408.

[9] N. V. Kuznetsov, G. A. Leonov, Counterexample of Perron in the Discrete Case, Izv. RAEN, Diff. Uravn., No 5, 2001, pp. 71.

[10] M. Bohner and A. Peterson, Dynamic equations on time scales: an introduction with applications, Birkhauser Boston, Inc., 2001.

[11] M. Bohner and A. Peterson, Advances in dynamic equations on time scales, Birkhauser Boston, Inc., Boston, 2003.

[12] J. Zhang, M. Fanb, H. Zhu, Necessary and sufficient criteria for the existence of exponential dichotomy on time scales, Computers and Mathematics with Applications 60 (2010) 2387-2398.

[13] A. Cabada and D. R. Vivero, Expression of the Lebesgue $\Delta$-integral on time scales as a usual Lebesgue integral; application to the calculus of $\Delta$-antiderivatives, Mathematical and Computer Modelling 43 (2006) 194-207. 